\title{
Weakly coupled de Sitter vacua with fluxes and the swampland
}

\section{Daniel Junghans}

Institut für Theoretische Physik, Ruprecht-Karls-Universität Heidelberg, Philosophenweg 19, Heidelberg 69120, Germany

E-mail: junghans@thphys.uni-heidelberg.de

ABSTRACT: It was recently argued that the swampland distance conjecture rules out dS vacua at parametrically large field distances. We point out that this conclusion can in principle be avoided in the presence of large fluxes that are not bounded by a tadpole cancellation condition. We then study this possibility in the concrete setting of classical type IIA flux compactifications with (anti-)O6-planes, (anti-)D6-branes and/or KK monopoles and show that, nonetheless, parametrically controlled dS vacua are strongly constrained. In particular, we find that such dS vacua are ruled out at parametrically large volume and/or parametrically small string coupling. We also find obstructions in the general case where the parametrically large field is an arbitrary field combination.

KEYWORDS: Flux compactifications, Superstring Vacua

ArXiv EPrint: 1811.06990 


\section{Contents}

1 Introduction 1

2 Avoiding the Dine-Seiberg runaway 3

3 Weakly coupled dS vacua in type IIA $\quad 6$

$\begin{array}{lll}3.1 \text { Large volume and/or small string coupling } & 6\end{array}$

3.2 Arbitrary field combination $\quad 9$

3.2.1 Argument 9

$\begin{array}{lll}3.2 .2 & \text { Caveats } & 11\end{array}$

4 Conclusions 13

\section{Introduction}

In recent years, a lot of effort has been devoted to constructing dS vacua in string theory. Starting with [1], many different scenarios and models have been proposed (see, e.g., [2$37])$. In order that all moduli are stabilized at a positive vacuum energy, these constructions require an intricate balance of several classical and quantum ingredients in the effective scalar potential. A possible concern with this approach is that the magnitude and moduli dependence of some of these ingredients and of possible corrections to them are not always known in full explicitness. While this may very well just be a technical issue, it has led some people to doubt the validity of the solutions. Furthermore, many no-go theorems rule out either the existence [38-55] or the stability [56-60] of dS extrema in various regions of the landscape. This has led to the interesting (although so far speculative) proposal that dS vacua might lie in the "swampland", i.e., that they are forbidden in quantum gravity (see, e.g., [61-63] and references therein and [64, 65] for different perspectives).

This general idea was recently further specified in [63] with the conjecture that an inequality $|\nabla V| \geq c V$ must hold everywhere in moduli space for some positive $\mathcal{O}(1)$ number $c$ (in Planck units). ${ }^{1}$ If true, this inequality would in particular exclude all dS critical points, regardless of whether they are maxima or minima. Since there appear to be a number of counter-examples [72-79], several authors subsequently proposed a refined version of the conjecture [80-82], which states that the inequality should only hold unless the minimal eigenvalue of the Hessian is bounded from above, $\min \left(\nabla_{i} \nabla_{j} V\right) \leq-c^{\prime} V$, where $c^{\prime}>0$ is another $\mathcal{O}(1)$ number (see also [83-85] for other proposals). Indeed, near no-scale points (which appear naturally in string theory), one can prove under mild assumptions that there is a universal tachyon at any dS critical point described by an F-term scalar potential [60]. This is consistent with the above conjecture for $c^{\prime}=\frac{4}{3}$. Nevertheless, it is currently far

\footnotetext{
${ }^{1}$ For recent discussions of dark energy and inflation in this context, see, e.g., [66-71].
} 
from clear whether something like a no-dS conspiracy can be expected to hold in string theory in general.

In the interesting recent paper [82], a step in this direction was taken by arguing that dS vacua are ruled out at parametrically large distances in moduli space. In order to show that, the authors used a general quantum gravity conjecture known as the swampland distance conjecture [86], together with an estimate of the dS entropy [87] based on the Bousso bound [88]. ${ }^{2}$ The swampland distance conjecture, while not proven, has been extensively studied in the recent literature and verified in various examples [86, 93-102]. In its refined version [94], it states that a tower of exponentially light states with masses $m \sim \mathrm{e}^{-\alpha \Delta \phi}$ (for some $\alpha>0$ ) should appear as we move a super-Planckian distance $\Delta \phi \gtrsim 1$ in field space (see also [103-105] for some caveats). This implies in particular that such a tower must appear at parametrically large field distances, $\phi \rightarrow \infty$. The states in the tower were found in [82] to lead to a dS entropy $S \sim \mathrm{e}^{a \phi}$ with $a>0$, which was then argued to imply a runaway in the scalar potential, $V \sim \mathrm{e}^{-a \phi}$. This result is reminiscent of an old argument due to Dine and Seiberg [106], where a runaway of this type was found for the case where $\phi$ is the dilaton. Another related argument, which bounds the potential from above by $\mathrm{e}^{-a \phi}$, was given in [107] based on an estimate of the cutoff scale in theories satisfying the swampland distance conjecture. In the same paper, it was pointed out that a potential of the type $V \sim n(\phi) \mathrm{e}^{-a \phi}$ with some oscillating function $n(\phi) \sim \mathcal{O}(1)$ is a priori not excluded by the entropy or cutoff arguments and could lead to wiggles stabilizing the runaway. Such an oscillating potential may in particular appear for axionic fields.

In this note, we point out a different loophole to the argument of [82] that exists in generic flux compactifications. In particular, we argue that, in the presence of fluxes that are unbounded by tadpole cancellation conditions, dS minima can in principle exist at parametrically large distances in moduli space without a runaway along the parametrically large field direction. We argue that this is possible without violating the swampland distance conjecture or the entropy bound, thus avoiding the no-go of [82].

One may wonder whether, in spite of this loophole, there is still a general obstruction to dS vacua at parametrically large field distances, perhaps due to some other reason unrelated to the above arguments. In fact, no parametrically controlled $\mathrm{dS}$ vacua have been constructed to date. While we do not present a general answer to this question, we analyze the possibility of parametrically controlled dS vacua in classical type IIA flux compactifications with (anti-)O6-planes, (anti-)D6-branes and/or KK monopoles. The possibility of dS vacua in this general class of compactifications was studied before in many papers $[37,41,43-45,58-60,74,85,108-112]$. We find that dS vacua are ruled out in this setting at parametrically large volume and/or parametrically small string coupling (with all other fields fixed). Our results are based on simple scaling arguments with respect to the moduli, similar to [41], and they do not assume the swampland distance conjecture. We also study the case where the parametrically large field is an arbitrary direction in field space and find strong constraints in this case as well.

\footnotetext{
${ }^{2}$ See also [89] for an argument relating the dS conjecture to the weak gravity conjecture [90] in a racetrack model and $[91,92]$ for arguments against dS vacua at the quantum level.
} 
Let us stress that going to parametrically large distances in field space need not be required to achieve perturbative control. Indeed, a dS minimum at, say, a volume $\mathcal{V}=10$ and a string coupling $g_{s}=0.1$ can already be very well-controlled as long as one can argue that corrections that are subleading in the $g_{s}$ and $1 / \mathcal{V}$ expansions have expansion coefficients $\ll \mathcal{O}(10)$. Nevertheless, it is interesting to contemplate a possible no-go against dS vacua at parametrically large field distances. For one thing, one may not always have full knowledge over the precise magnitude of subleading corrections but only know their scaling with respect to the expansion parameters. A parametric control over these corrections is then of course desirable. Second, in the spirit of the swampland program, it is worthwhile to understand possible universal properties of quantum gravity theories, including possible asymptotic properties. In this note, we will therefore only be concerned about statements at parametrically large field distances as in [82], keeping in mind that this does not rule out the existence of well-controlled dS vacua at moduli vevs which are large but not parametrically large. In the context of classical type IIA string theory, recent results [37, 112] suggest that dS minima might indeed be possible in such a regime. We will briefly comment on this possibility at the end of the paper.

This paper is organized as follows. In section 2, we review the Dine-Seiberg runaway, its relation to the entropy argument of [82] and how flux compactifications can avoid it. Crucially, we show that this is consistent with the swampland distance conjecture and the exponential entropy scaling derived in [82]. In section 3, we study our loophole in the context of classical type IIA flux compactifications with (anti-)O6-planes, (anti-)D6branes and/or KK monopoles. We find strong constraints on parametrically controlled dS vacua in this setting in spite of the presence of large fluxes. We conclude in section 4 with suggestions for future research directions and some comments on dS vacua without parametric control.

Note added. After completion of this work, we were informed about [113] which also discusses parametrically controlled dS vacua in type IIA string theory.

\section{Avoiding the Dine-Seiberg runaway}

Consider the scalar potential

$$
V(\phi)=A \mathrm{e}^{-a \phi}+B \mathrm{e}^{-(a+b) \phi}+C \mathrm{e}^{-(a+c) \phi}+\ldots,
$$

where $\phi$ is a canonically normalized modulus and $a>0, c>b>0, A, B, C$ are coefficients. The dots stand for possible other terms with a higher suppression with respect to $\phi$. In the limit

$$
\phi \rightarrow \infty, \quad A, B, C=\text { const. }
$$

only the first term in the potential survives,

$$
V(\phi) \sim \mathrm{e}^{-a \phi},
$$

such that no minima $\partial_{\phi} V=0$ are possible. In string compactifications, a potential of this type arises, for example, at weak string coupling (taking $\phi=-\ln \left(g_{s}\right)$ ) or at large volumes 
(taking $\phi \sim \ln \mathcal{V}$ with $\mathcal{V}=\int_{X} \mathrm{~d}^{6} y \sqrt{g_{6}}$ ). This is the well-known Dine-Seiberg problem [106]. It was originally discussed for the case where $\phi$ is the dilaton but analogous problems exist for other moduli (see, e.g., [114]). In particular, consider the volume modulus $\rho=\mathcal{V}^{1 / 3}$. In the limit $\rho \rightarrow \infty$, the potential is dominated by a leading term $V(\rho) \sim \rho^{-x}$ with $x>0$ such that no extrema can exist. Instead, the field either rolls towards a regime of small $\rho$ or there is a runaway to infinity. Dimensionally reducing the 10d Einstein-Hilbert term furthermore leads to a kinetic term of the form $\frac{(\partial \rho)^{2}}{\rho^{2}}$. The canonically normalized modulus is therefore $\phi \sim \ln \rho$ such that we recover the exponential behavior (2.3). In the following, we will refer to the limit (2.2) as the Dine-Seiberg regime, regardless of whether $\phi$ is the dilaton or some other field.

In [82], the behavior (2.3) was derived for arbitrary fields (for the case $V>0$ ) without requiring a concrete string theory input. Instead, the authors used a combination of the swampland distance conjecture and an entropy argument. They concluded that dS vacua are forbidden at asymptotic distances in moduli space. If true, this would in particular imply the intriguing result that dS vacua are forbidden at parametrically large volume and/or parametrically weak string coupling. However, we argue that this conclusion can be avoided in the presence of flux.

Indeed, it is well-known that, in flux compactifications $[114,115]$, the Dine-Seiberg runaway can be stabilized. We will see below that this is consistent with the entropy scaling derived in [82]. However, let us first review how the Dine-Seiberg problem is avoided in the presence of flux. The key point is that the coefficients $A, B, C$ are then not constants but depend on the flux numbers, which can be adjusted such that terms with a different $\phi$-scaling balance each other out even at parametrically large $\phi$. For example, the potential (2.1) admits dS minima at large $\phi$ for positive $A \sim \mathcal{O}(1)$, negative $B \sim \mathcal{O}\left(\mathrm{e}^{b \phi}\right)$ and positive $C \sim \mathcal{O}\left(\mathrm{e}^{c \phi}\right) .{ }^{3}$ In order to find minima with parametrically large $\phi$, the large- $\phi$ limit has to be taken such that

$$
\phi \rightarrow \infty, \quad A, B \mathrm{e}^{-b \phi}, C \mathrm{e}^{-c \phi}=\text { const. }
$$

More generally than (2.1), we could also consider potentials with more than three terms that all scale differently with respect to $\phi$ but are of the same order due to the flux dependence of their coefficients. In this regime, it is a priori not a problem to find minima at arbitrarily large $\phi$. It amounts to taking one or several fluxes parametrically large while approaching the asymptotic field distances. We will discuss this more explicitly in section 3 in the context of type IIA string theory. In the following, we will refer to this regime as the flux-balanced regime.

Typically, NSNS and RR fluxes are bounded by tadpole cancellation conditions of the form

$$
\frac{1}{\left(2 \pi \sqrt{\alpha^{\prime}}\right)^{1+p}} \int_{\Sigma} H_{3} \wedge F_{p}=Q^{\text {loc }}
$$

where the right-hand side stands for the charges of spacetime-filling branes and O-planes localized on a compact cycle $\Sigma$. This implies that the coefficients $B, C$ in (2.1) cannot

\footnotetext{
${ }^{3}$ A simple example is $A=\frac{7}{2}, B=-4 \lambda, C=\frac{3}{2} \lambda^{2}, a=b=1, c=2$, which yields a dS minimum at $\phi=\ln \lambda$ for arbitrarily large $\lambda$.
} 
be made arbitrarily large such that, for sufficiently large $\phi$, we inevitably end up in the Dine-Seiberg regime where no minima exist. However, there is no such bound whenever the fluxes arise from form fields whose legs are such that the $(3+p)$-form $H_{3} \wedge F_{p}$ vanishes on the compact space. ${ }^{4}$ A well-known example are the type IIA AdS vacua of [116], where minima at parametrically large volume and weak string coupling were found by taking the $F_{4}$ flux large. While this particular compactification does not admit dS vacua [41], other compactifications have not been ruled out this way. ${ }^{5}$

Let us now check that dS minima in the flux-balanced limit are consistent with the swampland distance conjecture. To see this, we briefly recall the argument of [82]. The swampland distance conjecture states that, for large $\phi$, there should be an exponentially light tower of states with masses $m \sim \mathrm{e}^{-\alpha \phi}$ for some coefficient $\alpha>0$. These light states contribute to the dS entropy $S$. Using the Bousso bound and assuming that the light states saturate the dS entropy for asymptotic values of $\phi$, it is then possible to show that

$$
S \sim \mathrm{e}^{a \phi}
$$

for some other coefficient $a>0$. Since the Gibbons-Hawking entropy of dS space [87] is inversely proportional to the vacuum energy, the authors of [82] concluded that the field dependence of the scalar potential must be $V \sim \mathrm{e}^{-a \phi}$ off-shell, leading to the runaway behavior (2.3) described above.

However, this need not be the case in the flux-balanced regime (2.4). In particular, the entropy scaling (2.6) constrains the magnitude, but not the off-shell field dependence, of the scalar potential to be $\sim \mathrm{e}^{-a \phi}$. Indeed, the entropy in the vicinity of the dS minimum described above is

$$
S \sim\left(A \mathrm{e}^{-a \phi}+B \mathrm{e}^{-(a+b) \phi}+C \mathrm{e}^{-(a+c) \phi}+\ldots\right)^{-1} \sim \mathrm{e}^{a \phi},
$$

where the right-hand side follows from the fact that, as we argued before, a flux choice $B \sim \mathrm{e}^{b \phi}, C \sim \mathrm{e}^{c \phi}$ is required at any minimum with parametrically large $\phi$. We thus recover the entropy scaling (2.6) predicted by the swampland distance conjecture. We conclude that the existence of dS minima at parametrically large field values does not seem to be in conflict with the swampland distance conjecture, at least not based on a pure counting of the entropy.

In [82], the authors impose the additional assumption that the entropy must increase strictly monotonically in the limit $\phi \rightarrow \infty$. If this is true, the flux loophole is ruled out. In particular, any displacement towards larger $\phi$ at fixed flux numbers would then lead to an increase of the entropy and, hence, a decrease of the potential, thus ruling out any minima. ${ }^{6}$

\footnotetext{
${ }^{4}$ In the presence of geometric fluxes, which are related to the Ricci curvature of the internal space, there can be additional contributions to the tadpole conditions that need to be taken into account.

${ }^{5}$ One can of course also find balanced potentials using quantum corrections (as, e.g., in [1, 3]). However, it is not obvious whether such potentials can admit terms with unbounded coefficients $B, C$. The clearest example for an unbounded coefficient seems to be provided by a flux term without a tadpole condition, so this is what we will focus on in this paper.

${ }^{6}$ Another loophole exploiting the possibility of a non-monotonic scalar potential is the "periodic-wiggle" loophole for axionic fields discussed in [107].
} 


\section{Weakly coupled dS vacua in type IIA}

If dS vacua at parametrically large field distances are in the swampland as proposed in [82], then we face a puzzle: why should there be no dS vacua in the flux-balanced regime (2.4), even though it appears to avoid a runaway problem? While we argued that the entropy scaling (2.6) does not in general rule out such vacua, they may still be forbidden for some other reason. In this section, we study this hypothesis in a class of string compactifications and indeed find obstructions to parametrically controlled dS vacua in spite of the loophole described above. Specifically, we consider classical type IIA flux compactifications with (anti-)O6-planes, (anti-)D6-branes and/or KK monopoles. This general class of compactifications has been studied before in many papers [37, 41, 43-45, 58-60, 74, 85, 108-112]. Recently, dS minima were found in this setup [37, 112], although so far at $\mathcal{O}(1)$ values for the volume and the dilaton and without properly quantized fluxes. ${ }^{7}$ As we will see in the following, it is impossible to construct similar dS minima at parametrically large volume and/or parametrically small string coupling (with all other fields fixed). We also study the general case where the parametrically large field is an arbitrary direction in field space and find strong constraints in this case as well.

\subsection{Large volume and/or small string coupling}

It is instructive to first discuss the case where the parametrically large field is the volume modulus, the dilaton or a combination of the two. This is arguably the most relevant case, as these two moduli are required to be large for perturbative control. We will later discuss a more general argument in section 3.2, which applies to any direction in field space.

The general $4 \mathrm{~d}$ scalar potential is [41]

$$
V(\tau, \rho)=-\frac{A_{\mathrm{O} 6 / \mathrm{D} 6}}{\rho^{9 / 2} \tau^{3}}+\sum_{p=0,2,4,6} \frac{A_{p}}{\rho^{3+p} \tau^{4}}+\frac{A_{3}}{\rho^{6} \tau^{2}}+\frac{A_{R}}{\rho^{4} \tau^{2}} .
$$

Here, we only display the dependence of the different terms with respect to the universal moduli

$$
\tau=\frac{1}{g_{s}}, \quad \rho=\mathcal{V}^{1 / 3},
$$

where $g_{s}=\mathrm{e}^{\phi}$ is the $10 \mathrm{~d}$ dilaton and $\mathcal{V}=\int_{X} \mathrm{~d}^{6} y \sqrt{g_{6}}$ is the string-frame volume. ${ }^{8}$ The coefficients $A_{i}=A_{i}\left(\varphi_{j}, n_{k}\right)$ depend on the other, non-universal moduli (such as cycle volumes or axions) and the flux numbers. They are obtained by dimensionally reducing the $10 \mathrm{~d}$ low-energy effective action of type IIA string theory. ${ }^{9}$ In particular, $A_{p}$ with $p$ even come from RR field strengths $\left|F_{p}\right|^{2}$ in the 10d action, $A_{3}$ comes from the NSNS field strength $\left|H_{3}\right|^{2}$ and $A_{R}$ comes from the internal Ricci scalar $R^{(6)}$. Note that $A_{R}$ may include

\footnotetext{
${ }^{7}$ The dS solutions were furthermore obtained in the smeared approximation (see, e.g., [117-123] for a discussion of this issue).

${ }^{8}$ Our moduli are related to those in [41] by $\rho=\rho_{[41]}$ and $\tau=\rho^{-3 / 2} \tau_{[41]}$.

${ }^{9}$ In the presence of localized sources such as O-planes or D-branes, compactifications are typically warped. While the resulting subtleties in the $4 \mathrm{~d}$ effective field theory are relatively well understood in type IIB/Ftheory (see, e.g., [124-137] and references therein), not much about this issue is known in type IIA. In the following, we ignore warping and assume that the $4 \mathrm{~d}$ scalar potential is well-approximated by (3.1).
} 
the effects of so-called geometric fluxes and/or KK monopoles, which have the same scaling with respect to the universal moduli (see, e.g., $[46,108]$ ). The $A_{\mathrm{O} 6 / \mathrm{D} 6}$-term comes from the DBI actions of (anti-)O6-planes and/or (anti-)D6-branes that wrap 3-cycles in the internal space. We refer to [41] for the details of the dimensional reduction and explicit expressions. For the following discussion, only two properties of these terms will be important:

- All coefficients are non-negative,

$$
A_{\mathrm{O} 6 / \mathrm{D} 6}, A_{R}>0, \quad A_{3}, A_{p} \geq 0 .
$$

For $A_{3}$ and $A_{p}$, this follows because the NSNS and RR field strengths are sums of squares. $A_{\mathrm{O} 6 / \mathrm{D} 6}$ could a priori be positive or negative, depending on whether it is dominated by the positive tension of branes $\left(A_{\mathrm{O} 6 / \mathrm{D} 6}<0\right)$ or the negative tension of O-planes $\left(A_{\mathrm{O} 6 / \mathrm{D} 6}>0\right)$. However, positivity here follows from the Maldacena-Nuñez no-go theorem [40], which states that a dS vacuum requires net negative tension, i.e., $A_{\mathrm{O} 6 / \mathrm{D} 6}>0$. Finally, it is known that a dS vacuum in the present setting requires negative internal curvature [41]. This corresponds to $A_{R}>0$.

- The coefficients $A_{3}, A_{p}$ and $A_{R}$ can be parametrically large due to their dependence on flux numbers. For example, in the simplest case of a single 4-cycle $\Sigma$ threaded by $F_{4}$-flux, we have

$$
A_{4} \sim n^{2}, \quad n=\frac{1}{\left(2 \pi \sqrt{\alpha^{\prime}}\right)^{3}} \int_{\Sigma} F_{4} .
$$

Here, $n$ can be a parametrically large number if $F_{4}$ is not bounded by a tadpole condition, as explained in section 2. Analogously, the coefficients $A_{3}, A_{p}$ and $A_{R}$ can in general be parametrically large due to their dependence on NSNS/RR fluxes and geometric fluxes/KK monopoles, respectively. Crucially, it is not possible for $A_{\mathrm{O} 6 / \mathrm{D} 6}$ to be parametrically large. One might think that one could make use of the fact that $A_{\mathrm{O} 6 / \mathrm{D} 6}$ depends on the brane and O-plane numbers. However, these are bounded due to the Maldacena-Nuñez theorem by which the O-plane contribution must dominate over the brane contribution. The O-plane number is given by the number of fixed points of the orientifold involution and is therefore finite and fixed in a given compactification.

For later convenience, we also state the equations of motion for $\rho$ and $\tau$. From (3.1), we find

$$
\begin{aligned}
& 0=\rho \partial_{\rho} V=\frac{9}{2} \frac{A_{\mathrm{O} 6 / \mathrm{D} 6}}{\rho^{9 / 2} \tau^{3}}-\sum_{p}(3+p) \frac{A_{p}}{\rho^{3+p} \tau^{4}}-6 \frac{A_{3}}{\rho^{6} \tau^{2}}-4 \frac{A_{R}}{\rho^{4} \tau^{2}}, \\
& 0=\tau \partial_{\tau} V=3 \frac{A_{\mathrm{O} 6 / \mathrm{D} 6}}{\rho^{9 / 2} \tau^{3}}-4 \sum_{p} \frac{A_{p}}{\rho^{3+p} \tau^{4}}-2 \frac{A_{3}}{\rho^{6} \tau^{2}}-2 \frac{A_{R}}{\rho^{4} \tau^{2}} .
\end{aligned}
$$

Our claim is now that the potential (3.1) does not admit any dS vacua at parametrically weak string coupling $(\tau \rightarrow \infty)$ and/or parametrically large volume $(\rho \rightarrow \infty)$. Let us first consider the simple case where, as in the AdS vacua of [116], the unbounded flux is $F_{4}$. We therefore study the behavior of (3.1) in the limit $\lambda \rightarrow \infty$ with $A_{4} \sim \lambda^{2}$ and $\rho \sim \lambda^{r}, \tau \sim \lambda^{t}$ 
for arbitrary exponents $r \geq 0, t \geq 0$. Note that we exclude negative exponents in order to avoid losing control. This yields the scalings

$$
\begin{aligned}
& \frac{A_{3}}{\rho^{6} \tau^{2}} \sim \lambda^{-6 r-2 t}, \quad \frac{A_{6}}{\rho^{9} \tau^{4}} \sim \lambda^{-9 r-4 t}, \quad \frac{A_{4}}{\rho^{7} \tau^{4}} \sim \lambda^{2-7 r-4 t}, \quad \frac{A_{2}}{\rho^{5} \tau^{4}} \sim \lambda^{-5 r-4 t}, \\
& \frac{A_{0}}{\rho^{3} \tau^{4}} \sim \lambda^{-3 r-4 t}, \quad \frac{A_{\mathrm{O} 6 / \mathrm{D} 6}}{\rho^{9 / 2} \tau^{3}} \sim \lambda^{-9 / 2 r-3 t}, \quad \frac{A_{R}}{\rho^{4} \tau^{2}} \sim \lambda^{-4 r-2 t} .
\end{aligned}
$$

Recall that, for a dS extremum to exist, we require both $A_{\mathrm{O} 6 / \mathrm{D} 6}>0$ and $A_{R}>0$. This corresponds to net negative tension [40] and negative internal curvature [41]. If the O6-plane term scales with a smaller power of $\lambda$ than the curvature term, it will be diluted away at large $\lambda$ and thus effectively vanish. The potential (3.1) then only has positive terms at leading order and yields a runaway. Hence, it is clear that dS vacua are forbidden unless we choose $r$ and $t$ such that the O6-plane term scales with the same or a higher power of $\lambda$ compared to the curvature term. Using (3.7), it then follows

$$
r \leq-2 t
$$

This is incompatible with the requirements $r \geq 0$ and $t \geq 0$ unless $r=t=0$ such that dS vacua are ruled out whenever $\tau$ and/or $\rho$ are parametrically large.

What if one of the other fluxes or several different fluxes are unbounded by a tadpole condition? We should then take into account the possibility of large fluxes $A_{p} \sim \lambda^{2 c_{p}}$, $A_{3} \sim \lambda^{2 c_{3}}$ for arbitrary exponents $c_{i} \geq 0$. However, it is clear that this does not affect the condition (3.8), which is due to the requirement that the $A_{\mathrm{O} 6 / \mathrm{D} 6}$-term is at least of the order of the $A_{R}$-term in (3.7). To be as general as possible, we can also consider a parametrically large coefficient $A_{R} \sim \lambda^{2 c_{R}}$ with $c_{R} \geq 0$. This could happen, e.g., due to large geometric fluxes or a large number of KK monopoles. We thus find

$$
\frac{A_{R}}{\rho^{4} \tau^{2}} \sim \lambda^{2 c_{R}-4 r-2 t}
$$

In order that the O6-plane term is not diluted away at large $\lambda$, we now require $r \leq-2 t-4 c_{R}$. This is again incompatible with $r \geq 0$ and $t \geq 0$ unless $r=t=c_{R}=0$.

The no-dS conclusion actually applies even more generally. For example, we can consider compactifications including (anti-)NS5-branes. This yields an additional term

$$
\frac{A_{\mathrm{NS} 5}}{\rho^{5} \tau^{2}} \sim \lambda^{c_{\mathrm{NS} 5}-5 r-2 t}
$$

in the scalar potential, where we admit a large number of NS5-branes for generality, i.e., $A_{\mathrm{NS} 5} \sim \lambda^{c_{\mathrm{NS} 5}}$ with $c_{\mathrm{NS} 5} \geq 0$. One can show that, in the presence of the NS5-branes, the requirement of negative internal curvature is dropped [41] such that a priori we need not run into the above difficulties. However, in order to allow dS minima for $\lambda \rightarrow \infty$, we now require that the O6-plane term is at least of the same order as the NS5-brane term. This is again due to our above argument that otherwise the O6-plane term would effectively vanish in the limit $\lambda \rightarrow \infty$. Hence, we have to consider $r \geq 2 c_{\mathrm{NS} 5}+2 t$. Substituting this into (3.7), we observe that the $A_{0}$ term now dominates over the $A_{\mathrm{O} 6 / \mathrm{D} 6}$ term at large 
$\lambda$-values. This is again an obstruction to dS vacua unless we either set $r=t=c_{\mathrm{NS} 5}=0$ or $A_{0}=0$. The potential in the latter case is

$$
V(\tau, \rho)=-\frac{A_{\mathrm{O} 6 / \mathrm{D} 6}}{\rho^{9 / 2} \tau^{3}}+\frac{A_{2}}{\rho^{5} \tau^{4}}+\frac{A_{4}}{\rho^{7} \tau^{4}}+\frac{A_{6}}{\rho^{9} \tau^{4}}+\frac{A_{3}}{\rho^{6} \tau^{2}}+\frac{A_{\mathrm{NS} 5}}{\rho^{5} \tau^{2}} .
$$

This satisfies $-\rho \partial_{\rho} V \geq \frac{9}{2} V$ and, hence, dS vacua are ruled out at parametrically large volume and/or parametrically weak string coupling even in the presence of NS5-branes.

\subsection{Arbitrary field combination}

Let us now discuss the general situation where the parametrically large field is any direction in field space. Indeed, one may wonder whether the above conclusion can be avoided if we exploit the fact that the coefficients $A_{i}$ can depend on non-universal moduli such as, e.g., 2-cycle or 3-cycle volumes. Instead of taking a limit where just a combination of $\rho$ and $\tau$ is parametrically large, one could try to also make some of these other moduli parametrically large or small in such a way that the scalings in (3.7) change. As we will explain in the following section 3.2.1, a rather suggestive argument rules out this idea under broad conditions. Our argument is, however, not waterproof, and we will discuss possible loopholes in section 3.2.2.

\subsubsection{Argument}

Let us consider some field $\alpha$, which is an arbitrary combination of the universal and nonuniversal moduli. We assume that $\alpha$ parametrizes some path in field space such that we are at a large distance from a strongly coupled point (i.e., a point with $\mathcal{O}(1)$ values for the volume and the string coupling) in the limit $\alpha \rightarrow \infty$.

The scalar potential is of the general form

$$
V(\alpha)=-\frac{\tilde{A}_{\mathrm{O} 6 / \mathrm{D} 6}}{\alpha^{x}}+\sum_{p} \frac{\tilde{A}_{p}(\alpha)}{\alpha^{x}}+\frac{\tilde{A}_{3}(\alpha)}{\alpha^{x}}+\frac{\tilde{A}_{R}(\alpha)}{\alpha^{x}}
$$

with $\tilde{A}_{\mathrm{O} 6 / \mathrm{D} 6}, \tilde{A}_{R}>0$ and $\tilde{A}_{p}, \tilde{A}_{3} \geq 0$ as before. ${ }^{10}$ Here, without loss of generality, we defined the field $\alpha$ such that the O6-plane term scales like $\alpha^{-x}$ in the limit $\alpha \rightarrow \infty$. In the following, we want to focus on a regime of parametric control, which implies that the energy densities in (3.12) should become small in the limit $\alpha \rightarrow \infty$. $^{11}$ The case $x<0$ is therefore excluded since then the energy densities diverge in the limit $\alpha \rightarrow \infty$ and we lose control. Similarly, we can neglect the case $x=0$ where the energy densities would approach constants instead of becoming small. For the remaining case $x>0$, we can then always set $x=1$ by a field redefinition. Also note that the $\alpha$-dependence of the terms other than the first one is so far completely general. We have only pulled out a common factor

\footnotetext{
${ }^{10}$ We indicate with a tilde that we pulled out a different field combination than in section 3.1.

${ }^{11}$ In particular, string-loop corrections are under parametric control if $g_{s}$ is parametrically small and $\alpha^{\prime}$ corrections are under parametric control if the string-frame energy densities in the 10d action are parametrically small. One can check that imposing either of the two implies that, after dimensional reduction and going to $4 \mathrm{~d}$ Einstein frame, the energy densities in the $4 \mathrm{~d}$ action are parametrically small.
} 
$\alpha^{-1}$ for later convenience. As previously, we do not explicitly write out the dependence of the terms on fields other than $\alpha$ and on the flux numbers.

Recall now that the O6-plane term must not be subleading for large $\alpha$. Crucially, this term does not depend on any flux numbers and does therefore not contain any parametrically large numbers that are not moduli. It is therefore not allowed to decay faster than the other terms in the limit $\alpha \rightarrow \infty$. This means that the functions $\tilde{A}_{i}$ are not allowed to diverge,

$$
\lim _{\alpha \rightarrow \infty} \tilde{A}_{i}(\alpha)<\infty \quad(i=0,2,3,4,6, R) .
$$

This would trivially be satisfied if all $\tilde{A}_{i}$ were constants in $\alpha$ but then the above potential would not yield any minima. However, the condition is also compatible with scalings such as, e.g., $\tilde{A}_{i} \sim \frac{n^{2}}{\alpha}$ or $\tilde{A}_{i} \sim n^{2} \mathrm{e}^{-\alpha}$. As explained before, the corresponding terms can then still contribute to the leading-order potential in the limit of large $\alpha$ if the flux $n$ is large.

Consider first the simplest possible case where all terms have a power-law scaling with respect to $\alpha$, i.e., $\tilde{A}_{i}(\alpha)=\tilde{B}_{i} / \alpha^{y_{i}}$. This yields the potential

$$
V(\alpha)=-\frac{\tilde{A}_{\mathrm{O} 6 / \mathrm{D} 6}}{\alpha}+\sum_{i} \frac{\tilde{B}_{i}}{\alpha^{1+y_{i}}}, \quad \tilde{B}_{i}, y_{i} \geq 0 .
$$

Solving the $\alpha$ equation for $\tilde{A}_{\mathrm{O} 6 / \mathrm{D} 6}$ and substituting this back into $V$, we find the on-shell potential

$$
V(\alpha)=-\sum_{i} \frac{y_{i} \tilde{B}_{i}}{\alpha^{1+y_{i}}}
$$

With $\tilde{B}_{i} \geq 0$ and $y_{i} \geq 0$, it follows that only AdS vacua are allowed. This is the essence of the problem with dS vacua at parametrically large field distances: the O6-plane term is the only negative term in the potential, and its coefficient cannot be made large. It must therefore be leading in the $\alpha$-expansion in order to contribute at large $\alpha$, which implies $\tilde{B}_{i} \geq 0$ and $y_{i} \geq 0$ for all $i$ and an absence of dS vacua.

Let us now analyze this problem for general $\tilde{A}_{i}$. The $\alpha$ equation is

$$
0=\frac{\tilde{A}_{\mathrm{O} 6 / \mathrm{D} 6}}{\alpha}-\sum_{p} \frac{\tilde{A}_{p}}{\alpha}-\frac{\tilde{A}_{3}}{\alpha}-\frac{\tilde{A}_{R}}{\alpha}+\sum_{p} \tilde{A}_{p}^{\prime}+\tilde{A}_{3}^{\prime}+\tilde{A}_{R}^{\prime},
$$

where $^{\prime}=\partial_{\alpha}$. Using (3.16) in (3.12), we can write the on-shell potential as

$$
0<V=\sum_{p} \tilde{A}_{p}^{\prime}+\tilde{A}_{3}^{\prime}+\tilde{A}_{R}^{\prime}
$$

where the right-hand side is strictly positive because $V>0$ at a dS vacuum. Using furthermore (3.16) in $V^{\prime \prime}$, we obtain the square of the $\alpha$-mass at the extremum, which needs to be positive:

$$
0<m_{\alpha}^{2}=V^{\prime \prime}=\sum_{p} \frac{\tilde{A}_{p}^{\prime \prime}}{\alpha}+\frac{\tilde{A}_{3}^{\prime \prime}}{\alpha}+\frac{\tilde{A}_{R}^{\prime \prime}}{\alpha} .
$$

Recall furthermore that all functions must satisfy $\tilde{A}_{i} \geq 0$. The key point is that these inequalities are difficult to satisfy while at the same time respecting that the functions 
should not diverge for large $\alpha$ (cf. (3.13)). For example, as discussed above, it is impossible to satisfy (3.17) for $\tilde{A}_{i} \sim \alpha^{-y_{i}}, y_{i}>0$ because then $\tilde{A}_{i}^{\prime}<0$. The same conclusion applies if $\tilde{A}_{i}$ is a sum of (positive) terms with such a scaling, or if we consider other decaying functions such as $\tilde{A}_{i} \sim \mathrm{e}^{-y_{i} \alpha}$ or $\tilde{A}_{i} \sim \ln (\alpha)^{-y_{i}}$. In any model with $\tilde{A}_{i}$ 's of this type, parametrically controlled dS solutions are ruled out. ${ }^{12}$

Some more care is required when some of the $\tilde{A}_{i}$ 's approach a constant at large $\alpha$, e.g., $\tilde{A}_{i} \sim n^{2} f(\alpha)$ with $f(\alpha)=c_{1}-\frac{c_{2}}{\alpha}+\ldots$ and $c_{1}, c_{2}$ some $\mathcal{O}(1)$ coefficients. For $c_{1}, c_{2}>0$, one can then satisfy $\tilde{A}_{i}>0$ and $\tilde{A}_{i}^{\prime}>0$ and thus potentially avoid the above arguments. Indeed, one checks that (3.17) and (3.18) can both be satisfied if at least one of the $\tilde{A}_{i}$ 's has a constant term, $c_{1} \neq 0$. However, these are only necessary conditions, and in fact one can show that such constant terms do not help to construct dS vacua either. ${ }^{13}$

We have thus seen that parametrically controlled dS vacua are ruled out under rather broad conditions, even if we allow the parametrically large field $\alpha$ to be an arbitrary field direction.

\subsubsection{Caveats}

In the remainder of this section, we will discuss several possible loopholes to these arguments. In particular, we focussed so far on $\tilde{A}_{i}$ 's which are dominated by one term with a unique scaling behavior at large $\alpha$. However, it could be that the above problems are avoided if more general functions $\tilde{A}_{i}$ are allowed where several terms with naively different scalings compete at large $\alpha$ due to parametrically large fluxes $n_{i}$. For example, consider

$$
\tilde{A}_{i} \sim c_{1} \frac{n_{1}^{2}}{\alpha^{y}}-c_{2} \frac{n_{1} n_{2}}{\alpha^{y+1}}+c_{3} \frac{n_{2}^{2}}{\alpha^{y+2}}+\ldots .
$$

One checks that both (3.17) and (3.18) can then be satisfied, and dS vacua can be obtained, if the fluxes $n_{i}$ and coefficients $c_{i}$ are such that the term in the middle is negative.

For the curvature term $\tilde{A}_{R}$, such a behavior is ruled out in many models even though $\tilde{A}_{R}$ may be a complicated function of the moduli and the fluxes in the interior of the moduli space. The reason is that, on many manifolds, the geometric fluxes cannot be made parametrically large because they are fixed by the geometry. At sufficiently large $\alpha, \tilde{A}_{R}$ is then inevitably dominated by a single term and thus satisfies a scaling law. For example, a well-studied class of negatively curved spaces are group manifolds, where the geometric

\footnotetext{
${ }^{12}$ In some models, the equations of motion admit dS solutions that have parametrically large 3-cycle volumes and thus formally avoid this conclusion, see, e.g., [58, 59]. However, these solutions either have parametrically small flux numbers or a parametrically large O6-plane number. This is not compatible with flux quantization and the fact that the number of orientifold fixed points is finite in a given compactification.

${ }^{13}$ To see this, notice that a subleading term $\sim c_{2}$ can only have an effect in (3.12) at large $\alpha$ if the leading terms in the potential cancel, i.e., $-\frac{\tilde{A}_{\mathrm{O} 6 / \mathrm{D} 6}}{\alpha}+\frac{n^{2} c_{1}}{\alpha}=0$. Since there are no parametrically large numbers in the coefficient $\tilde{A}_{\mathrm{O} 6 / \mathrm{D} 6}$, it then follows that the flux $n$ cannot be parametrically large either. The negative term $-\frac{n^{2} c_{2}}{\alpha^{2}}$ in $(3.12)$ thus has an $\mathcal{O}(1)$ coefficient, which means that it must be the leading term in the large- $\alpha$ expansion in (3.12) or else is negligible at large $\alpha$ (subleading terms could only be non-negligible if multiplied by parametrically large fluxes). We thus arrive at a potential where only the leading term in the $\alpha$ expansion is negative and all other relevant terms are positive (because all $\tilde{A}_{i}$ 's satisfy $\tilde{A}_{i} \geq 0$ ). One checks that such a potential cannot yield dS vacua, analogously to the situation discussed further above (e.g., around (3.14) and below).
} 
fluxes are the structure constants of the group and, hence, fixed $\mathcal{O}(1)$ numbers (see, e.g., [43, 111]). It would be interesting to study this further on other types of manifolds.

Similarly, $\tilde{A}_{3}$ and $\tilde{A}_{p}$ often have a simple scaling behavior at large $\alpha$ such that (3.19) is ruled out. In particular, they typically have the schematic structure (see, e.g., $[43,44,58]$ )

$$
\tilde{A}_{3}, \tilde{A}_{p} \sim f(\varphi)\left(n_{1}+n_{2} b+n_{3} b^{2}+n_{4} b^{3}\right)^{2}+\ldots,
$$

where $f(\varphi)$ is some a priori unknown function of all fields, $n_{i}$ are flux numbers and $b$ stands collectively for any axion. The dots denote further positive terms that have the same form as the displayed one. The number of terms appearing inside of the brackets depends on which of the $\tilde{A}_{i}$ we consider but this is irrelevant for our purpose. Consider now in particular the case where $\alpha$ is an arbitrary combination of non-axionic fields. The $\alpha$-dependence is then exclusively in the prefactor $f(\varphi)$. Hence, $\tilde{A}_{3}$ and $\tilde{A}_{p}$ are sums of positive terms which each have an overall scaling with respect to $\alpha$ in the limit $\alpha \rightarrow \infty$ and thus contribute negatively in (3.17).

A well-studied class of compactifications of type IIA string theory is on SU(3)-structure manifolds. The form (3.20) can then be shown to follow if the Kähler metric $K_{I J}$ is diagonal. This is true for some simple group and coset spaces considered in previous scans of classical dS vacua $[37,43-45,58,59,74,85,110-112]$. In general, however, the Kähler metric can have off-diagonal entries [43, 44], which would yield a structure $\tilde{A}_{i} \sim$ $K^{I J}(\varphi)\left(n_{I}+\ldots\right)\left(n_{J}+\ldots\right)$ instead of (3.20). The off-diagonal components of the metric can then produce $\alpha$-dependent terms that contribute with a minus sign as in (3.19) and thus in principle evade our argument. ${ }^{14}$ For example, in a simple two-field model and setting all axions to zero, we have $\tilde{A}_{i} \sim K^{11}(\varphi) n_{1}^{2}+K^{22}(\varphi) n_{2}^{2}+2 K^{12}(\varphi) n_{1} n_{2}$. For $K^{12}=0$, this is a sum of positive terms which depend on $\alpha$ only through the field-dependent prefactors $K^{11}$, $K^{22}$ such that the no-go argument of section 3.2.1 applies. On the other hand, considering a non-zero off-diagonal term $K^{12} \sim-\frac{1}{\alpha^{2}}$ together with $K^{11} \sim \frac{1}{\alpha}, K^{22} \sim \frac{1}{\alpha^{3}}$, we find an expression as in (3.19), and it may be possible to avoid the no-go. To see whether this works at large field distances, one would have to study negatively curved SU(3)-structure manifolds with sufficiently complicated moduli spaces, which we leave for future work.

Functions of the type (3.19) may also occur if one allows that $\alpha$ involves axionic fields, e.g., taking $b \sim \alpha$ in (3.20). This apparent possibility to avoid the above problems is not surprising given the axionic shift symmetries. In the presence of fluxes, axions acquire a potential that breaks these symmetries, but there is still a combined shift symmetry acting on the flux numbers and the axions together. For example, in the simple case $n_{3}=n_{4}=0,(3.20)$ is invariant under shifts $b \rightarrow b+\delta b, n_{1} \rightarrow n_{1}-n_{2} \delta b$. Therefore, whenever (3.1) admits a dS minimum at any $\mathcal{O}(1)$ values of the moduli, we can always map it to another dS minimum with formally large axion vevs by applying a shift. However, the large field value here is just an artifact of the parametrization, and we could always undo it by applying the symmetry. An analysis of whether dS vacua of (3.1) are excluded everywhere on the moduli space is beyond the scope of the current work, and we conclude that axions do not help to evade our argument.

\footnotetext{
${ }^{14}$ This conclusion cannot be avoided by rotating into a different basis with diagonal matrix $K^{I J}$, as the $n_{I}$ vectors only correspond to integer, field-independent flux numbers in one distinguished basis.
} 
There are some other caveats worth mentioning. First, the issues pointed out in this section are ameliorated if the $\tilde{A}_{i}$ are allowed to diverge in the limit $\alpha \rightarrow \infty$. A divergent $\tilde{A}_{i}$ may be possible if the function is proportional to a parameter which is not a modulus but can be taken parametrically small. For example, $\tilde{A}_{i} \sim \epsilon \alpha^{y}$ with $y>0$ and $\epsilon \rightarrow 0$ could satisfy (3.17), (3.18) and still be of the same order as the O6-plane term in (3.12). This is not possible for fluxes as they are quantized and can therefore at best be parametrically large. However, one might imagine, for example, a parametrically small warp factor appearing in some of the terms. Such a possibility was already mentioned in [41].

Second, an implicit assumption in our arguments was that all factors in the terms of the scalar potential have a well-defined asymptotic behavior in the large- $\alpha$ limit. This would not be satisfied, for example, for periodic factors such as $\cos (\alpha)$ (see also [107] for a discussion of such factors). While periodic functions are expected to occur along axionic field directions due to instanton corrections, it is not clear to us whether they could be relevant in the present setting where we considered a classical potential and its dependence on the non-axionic fields.

Let us finally point out that, although our result rules out dS vacua under the discussed assumptions, we do not know whether this implies the conjectured [63] property $|\nabla V| \geq$ $c V$ for some $\mathcal{O}(1)$ number $c>0$. The reason is that our argument does not make any assumptions about the explicit form of the kinetic term and the potential for the field $\alpha$. Therefore, we do not know how the potential scales with the corresponding canonically normalized field. In compactifications of the type studied in [37, 41, 43-45, 58-60, 74, 85, 108-112], the kinetic terms of the (non-axionic) moduli are of the form $(\partial \alpha)^{2} / \alpha^{2}$ and the potential is power-law. Any slope in the potential is therefore exponential in the canonically normalized field. In that case, an absence of dS extrema indeed implies that $|\nabla V| \geq c V$. However, our above argument does not prove this in general.

\section{Conclusions}

In this note, we pointed out a possible loophole to the recent argument [82] that dS minima are ruled out at parametrically large field distances. We argued that this conclusion can in principle be avoided in the presence of parametrically large flux. In particular, in the flux-balanced limit (2.4), dS vacua do not seem to be in contradiction with the swampland distance conjecture and the entropy scaling derived in [82].

We then analyzed whether our loophole allows to construct parametrically controlled dS vacua in the concrete setting of classical type IIA flux compactifications with (anti-)O6planes, (anti-)D6-branes and/or KK monopoles. We explicitly showed that such dS vacua are ruled out at parametrically large volume and/or parametrically small string coupling, and we found strong constraints in the general case where the parametrically large field is an arbitrary field combination.

We leave it as an exercise for future work to check whether similar statements are true for compactifications with more general ingredients such as, e.g., (anti-)O4-planes, (anti-)D4-branes or quantum corrections. It would also be interesting to investigate com- 
pactifications in type IIB string theory as in [46]. More ambitiously, one might attempt to formulate a general, model-independent argument (as in [82]) against parametrically controlled dS vacua in the flux-balanced regime.

Finally, we stress again that our argument has nothing to say about dS vacua at large volume and weak string coupling per se, as long as they are not under parametric control. For example, that dS minima might exist in the type IIA setting studied above is suggested by recent results that explain some of the difficulties in earlier attempts [58-60] and propose to avoid them using anti-D6-branes [37] or KK monopoles [112]. Indeed, simple dS minima, although so far not under perturbative control (i.e., not at large $\rho, \tau$ ), were constructed in $[37,112]$. It is at present not known whether there are similar minima also in the regime $\rho \gg 1, \tau \gg 1$. However, a simple scaling argument suggests that this might be the case.

To see this, recall the well-known fact that the type II string effective action at the classical two-derivative level has universal scaling symmetries [138, 139]. This implies, for example, that the scalar potential (3.1) is invariant (up to an overall factor) under the rescaling

$$
\rho \rightarrow \lambda^{2} \rho, \quad \tau \rightarrow \lambda \tau
$$

if we rescale at the same time the coefficients such that

$$
A_{\mathrm{O} 6 / \mathrm{D} 6} \rightarrow \lambda^{2} A_{\mathrm{O} 6 / \mathrm{D} 6}, \quad A_{p} \rightarrow \lambda^{2 p} A_{p}, \quad A_{3} \rightarrow \lambda^{4} A_{3}, \quad A_{R} \rightarrow A_{R} .
$$

Formally, any existing minimum in a given compactification can be mapped to a minimum at large volume and small coupling by the above rescaling. However, to reach large $\lambda$, we would need to rescale the flux numbers appearing in $A_{i}$ and the O6-plane number appearing in $A_{\mathrm{O} 6 / \mathrm{D} 6}$ (note that a large number of D6-branes alone would not help because then we would get $A_{\mathrm{O} 6 / \mathrm{D} 6}<0$ instead of the required $\left.A_{\mathrm{O} 6 / \mathrm{D} 6}>0\right)$. Since the O6plane number is fixed by the orientifold involution, we cannot take the limit $\lambda \rightarrow \infty$ in a given compactification and there is no parametric control. However, as discussed above, parametric control need not be required in order to argue for a dS minimum. Indeed, the scalings (4.2) suggest that compactifications with sufficiently many orientifold fixed points (i.e., with $\lambda \gg 1$ ) may admit minima analogous to those in $[37,112]$ at large volume and weak string coupling. It would be very interesting to study the mechanisms found in $[37,112]$ further on such orientifolds and check whether there are instabilities [140] or other obstructions to dS vacua there.

\section{Acknowledgments}

I would like to thank Arthur Hebecker, Gary Shiu, Pablo Soler, Thomas Van Riet and Timm Wrase for helpful discussions. I am supported in part by the DFG Transregional Collaborative Research Centre TRR 33 "The Dark Universe".

Open Access. This article is distributed under the terms of the Creative Commons Attribution License (CC-BY 4.0), which permits any use, distribution and reproduction in any medium, provided the original author(s) and source are credited. 


\section{References}

[1] S. Kachru, R. Kallosh, A.D. Linde and S.P. Trivedi, de Sitter vacua in string theory, Phys. Rev. D 68 (2003) 046005 [hep-th/0301240] [InSPIRE].

[2] C.P. Burgess, R. Kallosh and F. Quevedo, de Sitter string vacua from supersymmetric D terms, JHEP 10 (2003) 056 [hep-th/0309187] [INSPIRE].

[3] V. Balasubramanian, P. Berglund, J.P. Conlon and F. Quevedo, Systematics of moduli stabilisation in Calabi-Yau flux compactifications, JHEP 03 (2005) 007 [hep-th/0502058] [INSPIRE].

[4] M. Rummel and A. Westphal, A sufficient condition for de Sitter vacua in type IIB string theory, JHEP 01 (2012) 020 [arXiv:1107.2115] [INSPIRE].

[5] J. Louis, M. Rummel, R. Valandro and A. Westphal, Building an explicit de Sitter, JHEP 10 (2012) 163 [arXiv:1208.3208] [INSPIRE].

[6] M. Cicoli, A. Maharana, F. Quevedo and C.P. Burgess, de Sitter String Vacua from Dilaton-dependent Non-perturbative Effects, JHEP 06 (2012) 011 [arXiv:1203.1750] [INSPIRE].

[7] M. Cicoli, D. Klevers, S. Krippendorf, C. Mayrhofer, F. Quevedo and R. Valandro, Explicit de Sitter Flux Vacua for Global String Models with Chiral Matter, JHEP 05 (2014) 001 [arXiv: 1312.0014] [INSPIRE].

[8] J. Blåbäck, D. Roest and I. Zavala, de Sitter Vacua from Nonperturbative Flux Compactifications, Phys. Rev. D 90 (2014) 024065 [arXiv:1312.5328] [INSPIRE].

[9] U.H. Danielsson and G. Dibitetto, An alternative to anti-branes and O-planes?, JHEP 05 (2014) 013 [arXiv:1312.5331] [INSPIRE].

[10] M. Rummel and Y. Sumitomo, de Sitter Vacua from a D-term Generated Racetrack Uplift, JHEP 01 (2015) 015 [arXiv: 1407.7580] [InSPIRE].

[11] A.P. Braun, M. Rummel, Y. Sumitomo and R. Valandro, de Sitter vacua from a D-term generated racetrack potential in hypersurface Calabi-Yau compactifications, JHEP 12 (2015) 033 [arXiv:1509.06918] [INSPIRE].

[12] R. Kallosh, A.D. Linde, B. Vercnocke and T. Wrase, Analytic Classes of Metastable de Sitter Vacua, JHEP 10 (2014) 011 [arXiv:1406.4866] [INSPIRE].

[13] M.C.D. Marsh, B. Vercnocke and T. Wrase, Decoupling and de Sitter Vacua in Approximate No-Scale Supergravities, JHEP 05 (2015) 081 [arXiv:1411.6625] [INSPIRE].

[14] A. Guarino and G. Inverso, Single-step de Sitter vacua from nonperturbative effects with matter, Phys. Rev. D 93 (2016) 066013 [arXiv:1511.07841] [InSPIRE].

[15] A. Retolaza and A. Uranga, de Sitter Uplift with Dynamical SUSY Breaking, JHEP 04 (2016) 137 [arXiv:1512.06363] [INSPIRE].

[16] X. Dong, B. Horn, E. Silverstein and G. Torroba, Micromanaging de Sitter holography, Class. Quant. Grav. 27 (2010) 245020 [arXiv: 1005.5403] [INSPIRE].

[17] M. Dodelson, X. Dong, E. Silverstein and G. Torroba, New solutions with accelerated expansion in string theory, JHEP 12 (2014) 050 [arXiv:1310.5297] [INSPIRE].

[18] B. de Carlos, A. Guarino and J.M. Moreno, Flux moduli stabilisation, Supergravity algebras and no-go theorems, JHEP 01 (2010) 012 [arXiv:0907.5580] [INSPIRE]. 
[19] B. de Carlos, A. Guarino and J.M. Moreno, Complete classification of Minkowski vacua in generalised flux models, JHEP 02 (2010) 076 [arXiv:0911.2876] [INSPIRE].

[20] G. Dibitetto, R. Linares and D. Roest, Flux Compactifications, Gauge Algebras and de Sitter, Phys. Lett. B 688 (2010) 96 [arXiv:1001.3982] [InSPIRE].

[21] U.H. Danielsson and G. Dibitetto, On the distribution of stable de Sitter vacua, JHEP 03 (2013) 018 [arXiv:1212.4984] [INSPIRE].

[22] J. Blåbäck, U.H. Danielsson and G. Dibitetto, Fully stable dS vacua from generalised fluxes, JHEP 08 (2013) 054 [arXiv: 1301.7073] [InSPIRE].

[23] C. Damian, L.R. Diaz-Barron, O. Loaiza-Brito and M. Sabido, Slow-Roll Inflation in Non-geometric Flux Compactification, JHEP 06 (2013) 109 [arXiv:1302.0529] [INSPIRE].

[24] C. Damian and O. Loaiza-Brito, More stable de Sitter vacua from S-dual nongeometric fluxes, Phys. Rev. D 88 (2013) 046008 [arXiv: 1304.0792] [INSPIRE].

[25] F. Hassler, D. Lüst and S. Massai, On Inflation and de Sitter in Non-Geometric String Backgrounds, Fortsch. Phys. 65 (2017) 1700062 [arXiv:1405.2325] [INSPIRE].

[26] J. Blåbäck, U.H. Danielsson, G. Dibitetto and S.C. Vargas, Universal dS vacua in STU-models, JHEP 10 (2015) 069 [arXiv: 1505.04283] [INSPIRE].

[27] M. Cicoli, S. de Alwis and A. Westphal, Heterotic Moduli Stabilisation, JHEP 10 (2013) 199 [arXiv:1304.1809] [INSPIRE].

[28] S.L. Parameswaran and A. Westphal, de Sitter string vacua from perturbative Kähler corrections and consistent D-terms, JHEP 10 (2006) 079 [hep-th/0602253] [INSPIRE].

[29] C. Kounnas, D. Lüst and N. Toumbas, $R^{2}$ inflation from scale invariant supergravity and anomaly free superstrings with fluxes, Fortsch. Phys. 63 (2015) 12 [arXiv:1409.7076] [INSPIRE].

[30] A. Achúcarro, P. Ortiz and K. Sousa, A new class of de Sitter vacua in String Theory Compactifications, Phys. Rev. D 94 (2016) 086012 [arXiv:1510.01273] [INSPIRE].

[31] M. Cicoli, F. Quevedo and R. Valandro, de Sitter from T-branes, JHEP 03 (2016) 141 [arXiv: 1512.04558] [INSPIRE].

[32] R. Blumenhagen et al., A Flux-Scaling Scenario for High-Scale Moduli Stabilization in String Theory, Nucl. Phys. B 897 (2015) 500 [arXiv:1503.07634] [inSPIRE].

[33] R. Blumenhagen, C. Damian, A. Font, D. Herschmann and R. Sun, The Flux-Scaling Scenario: de Sitter Uplift and Axion Inflation, Fortsch. Phys. 64 (2016) 536 [arXiv: 1510.01522] [INSPIRE].

[34] D. Gallego, M.C.D. Marsh, B. Vercnocke and T. Wrase, A New Class of de Sitter Vacua in Type IIB Large Volume Compactifications, JHEP 10 (2017) 193 [arXiv:1707.01095] [INSPIRE].

[35] T. Kobayashi, N. Omoto, H. Otsuka and T.H. Tatsuishi, Radiative Kähler moduli stabilization, Phys. Rev. D 97 (2018) 106006 [arXiv:1711.10274] [INSPIRE].

[36] I. Antoniadis, Y. Chen and G.K. Leontaris, Perturbative moduli stabilisation in type IIB/F-theory framework, Eur. Phys. J. C 78 (2018) 766 [arXiv:1803.08941] [INSPIRE].

[37] R. Kallosh and T. Wrase, dS Supergravity from 10d, Fortsch. Phys. 67 (2019) 1800071 [arXiv: 1808.09427] [INSPIRE]. 
[38] G.W. Gibbons, Aspects of Supergravity Theories, in Supersymmetry, Supergravity and Related Topics, F. del Aguila, J.A. de Azcárraga and L.E. Ibáñez eds., World Scientific (1985), pp. 346-351.

[39] B. de Wit, D.J. Smit and N.D. Hari Dass, Residual Supersymmetry of Compactified D $=10$ Supergravity, Nucl. Phys. B 283 (1987) 165 [InSPIRE].

[40] J.M. Maldacena and C. Núñez, Supergravity description of field theories on curved manifolds and a no go theorem, Int. J. Mod. Phys. A 16 (2001) 822 [hep-th/0007018] [INSPIRE].

[41] M.P. Hertzberg, S. Kachru, W. Taylor and M. Tegmark, Inflationary Constraints on Type IIA String Theory, JHEP 12 (2007) 095 [arXiv:0711.2512] [INSPIRE].

[42] P.J. Steinhardt and D. Wesley, Dark Energy, Inflation and Extra Dimensions, Phys. Rev. D 79 (2009) 104026 [arXiv:0811.1614] [InSPIRE].

[43] C. Caviezel, P. Koerber, S. Körs, D. Lüst, T. Wrase and M. Zagermann, On the Cosmology of Type IIA Compactifications on SU(3)-structure Manifolds, JHEP 04 (2009) 010 [arXiv:0812.3551] [INSPIRE].

[44] R. Flauger, S. Paban, D. Robbins and T. Wrase, Searching for slow-roll moduli inflation in massive type IIA supergravity with metric fluxes, Phys. Rev. D 79 (2009) 086011 [arXiv:0812.3886] [INSPIRE].

[45] U.H. Danielsson, S.S. Haque, G. Shiu and T. Van Riet, Towards Classical de Sitter Solutions in String Theory, JHEP 09 (2009) 114 [arXiv:0907.2041] [INSPIRE].

[46] C. Caviezel, T. Wrase and M. Zagermann, Moduli Stabilization and Cosmology of Type IIB on SU(2)-Structure Orientifolds, JHEP 04 (2010) 011 [arXiv:0912.3287] [INSPIRE].

[47] T. Wrase and M. Zagermann, On Classical de Sitter Vacua in String Theory, Fortsch. Phys. 58 (2010) 906 [arXiv: 1003.0029] [INSPIRE].

[48] T. Van Riet, On classical de Sitter solutions in higher dimensions, Class. Quant. Grav. 29 (2012) 055001 [arXiv:1111.3154] [INSPIRE].

[49] S.R. Green, E.J. Martinec, C. Quigley and S. Sethi, Constraints on String Cosmology, Class. Quant. Grav. 29 (2012) 075006 [arXiv:1110.0545] [INSPIRE].

[50] F.F. Gautason, D. Junghans and M. Zagermann, On Cosmological Constants from $\alpha^{\prime}$-Corrections, JHEP 06 (2012) 029 [arXiv:1204.0807] [INSPIRE].

[51] D. Kutasov, T. Maxfield, I. Melnikov and S. Sethi, Constraining de Sitter Space in String Theory, Phys. Rev. Lett. 115 (2015) 071305 [arXiv:1504.00056] [INSPIRE].

[52] C. Quigley, Gaugino Condensation and the Cosmological Constant, JHEP 06 (2015) 104 [arXiv: 1504.00652] [INSPIRE].

[53] D. Andriot and J. Blåbäck, Refining the boundaries of the classical de Sitter landscape, JHEP 03 (2017) 102 [Erratum JHEP 03 (2018) 083] [arXiv:1609.00385] [INSPIRE].

[54] D. Andriot, On classical de Sitter and Minkowski solutions with intersecting branes, JHEP 03 (2018) 054 [arXiv: 1710.08886] [INSPIRE].

[55] D. Andriot, New constraints on classical de Sitter: flirting with the swampland, Fortsch. Phys. 67 (2019) 1800103 [arXiv:1807.09698] [INSPIRE].

[56] L. Covi, M. Gomez-Reino, C. Gross, J. Louis, G.A. Palma and C.A. Scrucca, de Sitter vacua in no-scale supergravities and Calabi-Yau string models, JHEP 06 (2008) 057 [arXiv: 0804.1073] [INSPIRE]. 
[57] G. Shiu and Y. Sumitomo, Stability Constraints on Classical de Sitter Vacua, JHEP 09 (2011) 052 [arXiv:1107.2925] [INSPIRE].

[58] U.H. Danielsson, G. Shiu, T. Van Riet and T. Wrase, A note on obstinate tachyons in classical dS solutions, JHEP 03 (2013) 138 [arXiv:1212.5178] [INSPIRE].

[59] D. Junghans, Tachyons in Classical de Sitter Vacua, JHEP 06 (2016) 132 [arXiv: 1603.08939] [INSPIRE].

[60] D. Junghans and M. Zagermann, A Universal Tachyon in Nearly No-scale de Sitter Compactifications, JHEP 07 (2018) 078 [arXiv: 1612.06847] [INSPIRE].

[61] T.D. Brennan, F. Carta and C. Vafa, The String Landscape, the Swampland and the Missing Corner, PoS(TASI2017)015 (2017) [arXiv:1711.00864] [INSPIRE].

[62] U.H. Danielsson and T. Van Riet, What if string theory has no de Sitter vacua?, Int. J. Mod. Phys. D 27 (2018) 1830007 [arXiv: 1804.01120] [InSPIRE].

[63] G. Obied, H. Ooguri, L. Spodyneiko and C. Vafa, de Sitter Space and the Swampland, arXiv: 1806.08362 [INSPIRE].

[64] M. Cicoli, S. de Alwis, A. Maharana, F. Muia and F. Quevedo, de Sitter vs. Quintessence in String Theory, Fortsch. Phys. 67 (2019) 1800079 [arXiv:1808.08967] [INSPIRE].

[65] Y. Akrami, R. Kallosh, A.D. Linde and V. Vardanyan, The Landscape, the Swampland and the Era of Precision Cosmology, Fortsch. Phys. 67 (2019) 1800075 [arXiv:1808.09440] [INSPIRE].

[66] E.Ó. Colgáin, M.H.P.M. Van Putten and H. Yavartanoo, Observational consequences of $H_{0}$ tension in de Sitter Swampland, arXiv: 1807.07451 [INSPIRE].

[67] L. Heisenberg, M. Bartelmann, R. Brandenberger and A. Refregier, Dark Energy in the Swampland, Phys. Rev. D 98 (2018) 123502 [arXiv:1808.02877] [INSPIRE].

[68] C. Damian and O. Loaiza-Brito, Two-Field Axion Inflation and the Swampland Constraint in the Flux-Scaling Scenario, Fortsch. Phys. 67 (2019) 1800072 [arXiv:1808.03397] [INSPIRE].

[69] C. Han, S. Pi and M. Sasaki, Quintessence Saves Higgs Instability, arXiv:1809.05507 [INSPIRE].

[70] A. Ashoorioon, Rescuing Single Field Inflation from the Swampland, Phys. Lett. B $\mathbf{7 9 0}$ (2019) 568 [arXiv:1810.04001] [INSPIRE].

[71] J.J. Heckman, C. Lawrie, L. Lin and G. Zoccarato, F-theory and Dark Energy, arXiv: 1811.01959 [INSPIRE].

[72] F. Denef, A. Hebecker and T. Wrase, de Sitter swampland conjecture and the Higgs potential, Phys. Rev. D 98 (2018) 086004 [arXiv:1807.06581] [InSPIRE].

[73] J.P. Conlon, The de Sitter swampland conjecture and supersymmetric AdS vacua, Int. J. Mod. Phys. A 33 (2018) 1850178 [arXiv: 1808.05040] [InSPIRE].

[74] C. Roupec and T. Wrase, de Sitter Extrema and the Swampland, Fortsch. Phys. 67 (2019) 1800082 [arXiv:1807.09538] [INSPIRE].

[75] H. Murayama, M. Yamazaki and T.T. Yanagida, Do We Live in the Swampland?, JHEP 12 (2018) 032 [arXiv:1809.00478] [INSPIRE]. 
[76] K. Choi, D. Chway and C.S. Shin, The dS swampland conjecture with the electroweak symmetry and QCD chiral symmetry breaking, JHEP 11 (2018) 142 [arXiv:1809.01475] [INSPIRE].

[77] K. Hamaguchi, M. Ibe and T. Moroi, The swampland conjecture and the Higgs expectation value, JHEP 12 (2018) 023 [arXiv:1810.02095] [INSPIRE].

[78] Y. Olguín-Trejo, S.L. Parameswaran, G. Tasinato and I. Zavala, Runaway Quintessence, Out of the Swampland, JCAP 01 (2019) 031 [arXiv: 1810.08634] [INSPIRE].

[79] J.J. Blanco-Pillado, M.A. Urkiola and J.M. Wachter, Racetrack Potentials and the de Sitter Swampland Conjectures, JHEP 01 (2019) 187 [arXiv:1811.05463] [INSPIRE].

[80] D. Andriot, On the de Sitter swampland criterion, Phys. Lett. B 785 (2018) 570 [arXiv:1806.10999] [INSPIRE].

[81] S.K. Garg and C. Krishnan, Bounds on Slow Roll and the de Sitter Swampland, arXiv: 1807.05193 [INSPIRE].

[82] H. Ooguri, E. Palti, G. Shiu and C. Vafa, Distance and de Sitter Conjectures on the Swampland, Phys. Lett. B 788 (2019) 180 [arXiv:1810.05506] [INSPIRE].

[83] G. Dvali and C. Gomez, On Exclusion of Positive Cosmological Constant, Fortsch. Phys. 67 (2019) 1800092 [arXiv:1806.10877] [INSPIRE].

[84] G. Dvali, C. Gomez and S. Zell, Quantum Breaking Bound on de Sitter and Swampland, Fortsch. Phys. 67 (2019) 1800094 [arXiv:1810.11002] [InSPIRE].

[85] S.K. Garg, C. Krishnan and M. Zaid Zaz, Bounds on Slow Roll at the Boundary of the Landscape, JHEP 03 (2019) 029 [arXiv: 1810.09406] [INSPIRE].

[86] H. Ooguri and C. Vafa, On the Geometry of the String Landscape and the Swampland, Nucl. Phys. B 766 (2007) 21 [hep-th/0605264] [INSPIRE].

[87] G.W. Gibbons and S.W. Hawking, Cosmological Event Horizons, Thermodynamics and Particle Creation, Phys. Rev. D 15 (1977) 2738 [InSPIRE].

[88] R. Bousso, A Covariant entropy conjecture, JHEP 07 (1999) 004 [hep-th/9905177] [INSPIRE].

[89] J. Moritz and T. Van Riet, Racing through the swampland: de Sitter uplift vs. weak gravity, JHEP 09 (2018) 099 [arXiv: 1805.00944] [INSPIRE].

[90] N. Arkani-Hamed, L. Motl, A. Nicolis and C. Vafa, The String landscape, black holes and gravity as the weakest force, JHEP 06 (2007) 060 [hep-th/0601001] [InSPIRE].

[91] K. Dasgupta, M. Emelin, E. McDonough and R. Tatar, Quantum Corrections and the de Sitter Swampland Conjecture, JHEP 01 (2019) 145 [arXiv:1808.07498] [INSPIRE].

[92] U.H. Danielsson, The quantum swampland, arXiv:1809.04512 [INSPIRE].

[93] F. Baume and E. Palti, Backreacted Axion Field Ranges in String Theory, JHEP 08 (2016) 043 [arXiv:1602.06517] [INSPIRE].

[94] D. Kläwer and E. Palti, Super-Planckian Spatial Field Variations and Quantum Gravity, JHEP 01 (2017) 088 [arXiv: 1610.00010] [INSPIRE].

[95] I. Valenzuela, Backreaction Issues in Axion Monodromy and Minkowski 4-forms, JHEP 06 (2017) 098 [arXiv: 1611.00394] [INSPIRE]. 
[96] R. Blumenhagen, I. Valenzuela and F. Wolf, The Swampland Conjecture and F-term Axion Monodromy Inflation, JHEP 07 (2017) 145 [arXiv: 1703.05776] [INSPIRE].

[97] E. Palti, The Weak Gravity Conjecture and Scalar Fields, JHEP 08 (2017) 034 [arXiv: 1705.04328] [INSPIRE].

[98] M. Cicoli, D. Ciupke, C. Mayrhofer and P. Shukla, A Geometrical Upper Bound on the Inflaton Range, JHEP 05 (2018) 001 [arXiv: 1801.05434] [INSPIRE].

[99] T.W. Grimm, E. Palti and I. Valenzuela, Infinite Distances in Field Space and Massless Towers of States, JHEP 08 (2018) 143 [arXiv: 1802.08264] [INSPIRE].

[100] B. Heidenreich, M. Reece and T. Rudelius, Emergence of Weak Coupling at Large Distance in Quantum Gravity, Phys. Rev. Lett. 121 (2018) 051601 [arXiv: 1802.08698] [INSPIRE].

[101] R. Blumenhagen, D. Kläwer, L. Schlechter and F. Wolf, The Refined Swampland Distance Conjecture in Calabi-Yau Moduli Spaces, JHEP 06 (2018) 052 [arXiv:1803.04989] [INSPIRE].

[102] T.W. Grimm, C. Li and E. Palti, Infinite Distance Networks in Field Space and Charge Orbits, JHEP 03 (2019) 016 [arXiv:1811.02571] [INSPIRE].

[103] A. Hebecker, P. Henkenjohann and L.T. Witkowski, Flat Monodromies and a Moduli Space Size Conjecture, JHEP 12 (2017) 033 [arXiv:1708.06761] [INSPIRE].

[104] A. Landete and G. Shiu, Mass Hierarchies and Dynamical Field Range, Phys. Rev. D 98 (2018) 066012 [arXiv:1806.01874] [InSPIRE].

[105] A. Hebecker, D. Junghans and A. Schachner, Large Field Ranges from Aligned and Misaligned Winding, arXiv:1812.05626 [INSPIRE].

[106] M. Dine and N. Seiberg, Is the Superstring Weakly Coupled?, Phys. Lett. B 162 (1985) 299 [INSPIRE].

[107] A. Hebecker and T. Wrase, The Asymptotic dS Swampland Conjecture - a Simplified Derivation and a Potential Loophole, Fortsch. Phys. 67 (2019) 1800097 [arXiv: 1810.08182] [INSPIRE].

[108] E. Silverstein, Simple de Sitter Solutions, Phys. Rev. D 77 (2008) 106006 [arXiv:0712.1196] [INSPIRE].

[109] S.S. Haque, G. Shiu, B. Underwood and T. Van Riet, Minimal simple de Sitter solutions, Phys. Rev. D 79 (2009) 086005 [arXiv:0810.5328] [InSPIRE].

[110] U.H. Danielsson, P. Koerber and T. Van Riet, Universal de Sitter solutions at tree-level, JHEP 05 (2010) 090 [arXiv: 1003.3590] [INSPIRE].

[111] U.H. Danielsson, S.S. Haque, P. Koerber, G. Shiu, T. Van Riet and T. Wrase, de Sitter hunting in a classical landscape, Fortsch. Phys. 59 (2011) 897 [arXiv:1103.4858] [INSPIRE].

[112] J. Blåbäck, U.H. Danielsson and G. Dibitetto, A new light on the darkest corner of the landscape, arXiv: 1810.11365 [INSPIRE].

[113] A. Banlaki, A. Chowdhury, C. Roupec and T. Wrase, Scaling limits of dS vacua and the swampland, arXiv:1811.07880 [INSPIRE].

[114] F. Denef, Les Houches Lectures on Constructing String Vacua, Les Houches 87 (2008) 483 [arXiv: 0803.1194] [INSPIRE]. 
[115] M.R. Douglas and S. Kachru, Flux compactification, Rev. Mod. Phys. 79 (2007) 733 [hep-th/0610102] [INSPIRE].

[116] O. DeWolfe, A. Giryavets, S. Kachru and W. Taylor, Type IIA moduli stabilization, JHEP 07 (2005) 066 [hep-th/0505160] [INSPIRE].

[117] M.R. Douglas and R. Kallosh, Compactification on negatively curved manifolds, JHEP 06 (2010) 004 [arXiv: 1001.4008] [INSPIRE].

[118] J. Blåbäck, U.H. Danielsson, D. Junghans, T. Van Riet, T. Wrase and M. Zagermann, Smeared versus localised sources in flux compactifications, JHEP 12 (2010) 043 [arXiv: 1009.1877] [INSPIRE].

[119] J. Blåbäck, U.H. Danielsson, D. Junghans, T. Van Riet, T. Wrase and M. Zagermann, The problematic backreaction of SUSY-breaking branes, JHEP 08 (2011) 105

[arXiv:1105.4879] [INSPIRE].

[120] J. Blåbäck, U.H. Danielsson, D. Junghans, T. Van Riet, T. Wrase and M. Zagermann, (Anti-)Brane backreaction beyond perturbation theory, JHEP 02 (2012) 025 [arXiv: 1111.2605] [INSPIRE].

[121] F. Saracco and A. Tomasiello, Localized O6-plane solutions with Romans mass, JHEP 07 (2012) 077 [arXiv:1201.5378] [InSPIRE].

[122] J. McOrist and S. Sethi, M-theory and Type IIA Flux Compactifications, JHEP 12 (2012) 122 [arXiv:1208.0261] [INSPIRE].

[123] F.F. Gautason, M. Schillo, T. Van Riet and M. Williams, Remarks on scale separation in flux vacua, JHEP 03 (2016) 061 [arXiv: 1512.00457] [INSPIRE].

[124] O. DeWolfe and S.B. Giddings, Scales and hierarchies in warped compactifications and brane worlds, Phys. Rev. D 67 (2003) 066008 [hep-th/0208123] [INSPIRE].

[125] S.B. Giddings and A. Maharana, Dynamics of warped compactifications and the shape of the warped landscape, Phys. Rev. D 73 (2006) 126003 [hep-th/0507158] [INSPIRE].

[126] A.R. Frey and A. Maharana, Warped spectroscopy: Localization of frozen bulk modes, JHEP 08 (2006) 021 [hep-th/0603233] [INSPIRE].

[127] P. Koerber and L. Martucci, From ten to four and back again: How to generalize the geometry, JHEP 08 (2007) 059 [arXiv:0707.1038] [INSPIRE].

[128] G. Shiu, G. Torroba, B. Underwood and M.R. Douglas, Dynamics of Warped Flux Compactifications, JHEP 06 (2008) 024 [arXiv:0803.3068] [INSPIRE].

[129] M.R. Douglas and G. Torroba, Kinetic terms in warped compactifications, JHEP 05 (2009) 013 [arXiv:0805.3700] [INSPIRE].

[130] A.R. Frey, G. Torroba, B. Underwood and M.R. Douglas, The Universal Kähler Modulus in Warped Compactifications, JHEP 01 (2009) 036 [arXiv:0810.5768] [INSPIRE].

[131] L. Martucci, On moduli and effective theory of $N=1$ warped flux compactifications, JHEP 05 (2009) 027 [arXiv:0902.4031] [INSPIRE].

[132] B. Underwood, A Breathing Mode for Warped Compactifications, Class. Quant. Grav. 28 (2011) 195013 [arXiv: 1009.4200] [INSPIRE].

[133] A.R. Frey and J. Roberts, The Dimensional Reduction and Kähler Metric of Forms In Flux and Warping, JHEP 10 (2013) 021 [arXiv:1308.0323] [INSPIRE]. 
[134] L. Martucci, Warping the Kähler potential of F-theory/IIB flux compactifications, JHEP 03 (2015) 067 [arXiv: 1411.2623] [INSPIRE].

[135] T.W. Grimm, T.G. Pugh and M. Weissenbacher, The effective action of warped M-theory reductions with higher derivative terms. Part I, JHEP 01 (2016) 142 [arXiv:1412.5073] [INSPIRE].

[136] T.W. Grimm, T.G. Pugh and M. Weissenbacher, The effective action of warped M-theory reductions with higher-derivative terms. Part II, JHEP 12 (2015) 117 [arXiv:1507.00343] [INSPIRE].

[137] L. Martucci, Warped Kähler potentials and fluxes, JHEP 01 (2017) 056 [arXiv: 1610.02403] [INSPIRE].

[138] E. Witten, Dimensional Reduction of Superstring Models, Phys. Lett. B 155 (1985) 151 [INSPIRE].

[139] C.P. Burgess, A. Font and F. Quevedo, Low-Energy Effective Action for the Superstring, Nucl. Phys. B 272 (1986) 661 [inSPIRE].

[140] U.H. Danielsson, F.F. Gautason and T. Van Riet, Unstoppable brane-flux decay of $\overline{\mathrm{D} 6}$ branes, JHEP 03 (2017) 141 [arXiv: 1609.06529] [INSPIRE]. 